\title{
Low-redshift formula for the luminosity distance in a LTB model with cosmological constant
}

\author{
Antonio Enea Romano ${ }^{1,3,4, a}$, Pisin Chen ${ }^{1,2, b}$ \\ ${ }^{1}$ Leung Center for Cosmology and Particle Astrophysics, National Taiwan University, Taipei, Taiwan \\ ${ }^{2}$ Kavli Institute for Particle Astrophysics and Cosmology, SLAC National Accelerator Laboratory, Menlo Park, CA 94025, USA \\ ${ }^{3}$ Yukawa Institute for Theoretical Physics, Kyoto University, Kyoto 606-8502, Japan \\ ${ }^{4}$ Instituto de Fisica, Universidad de Antioquia, A.A.1226 Medellin, Colombia
}

Received: 29 August 2013 / Accepted: 14 February 2014 / Published online: 1 April 2014

(C) The Author(s) 2014. This article is published with open access at Springerlink.com

\begin{abstract}
We calculate the low-redshift Taylor expansion for the luminosity distance for an observer at the center of a spherically symmetric matter inhomogeneity with a nonvanishing cosmological constant. We then test the accuracy of the formulas comparing them to the numerical calculation for different cases for both the luminosity distance and the radial coordinate. The formulas can be used as a starting point to understand the general non-linear effects of a local inhomogeneity in the presence of a cosmological constant, without making any special assumption as regards the inhomogeneity profile.
\end{abstract}

\section{Introduction}

Modern cosmological observations such as the luminosity distance [1-6] and the WMAP measurements $[7,8]$ of the cosmic microwave background radiation (CMBR) have provided strong evidence for the presence of dark energy. One of the main assumptions of the standard cosmological model used in fitting these observational data is spatial homogeneity of the Universe. We cannot nevertheless exclude the presence of a local inhomogeneity around us which could affect our interpretation of the cosmological data [9-11].

So far most of the efforts in estimating these effects have consisted in using some ansatz for the profile of the inhomogeneity and then calculating numerically the effects on cosmological observables. Such an approach has the limitation of depending on the particular functional form chosen to model the local inhomogeneity, and of relying completely on numerical calculations. In order to provide a more general study of this effects we approach the problem analytically and

\footnotetext{
a e-mail: aer@phys.ntu.edu.tw

b e-mail: pisinchen@phys.ntu.edu.tw
}

we derive a low-redshift formula for the luminosity distance for an observer at the center of a matter inhomogeneity in the presence of a cosmological constant modeled by a LTB solution.

The paper is organized as follows. We first calculate the low-redshift expansion of the null radial geodesics for a central observer and then use it to obtain the luminosity distance. The calculation is based on using the analytical solution and the geodesic equation expressed in the same coordinates of the analytical solution. The formula obtained is then compared to the numerical calculation of the luminosity distance to test its accuracy. In the appendix we give details of the derivation and the simplified formulas in the limit in which the inhomogeneity can be treated perturbatively.

\section{LTB solution with a cosmological constant}

The LTB solution can be expressed in the form [12-14]

$\mathrm{d} s^{2}=-\mathrm{d} t^{2}+\frac{(R, r)^{2} \mathrm{~d} r^{2}}{1+2 E(r)}+R^{2} \mathrm{~d} \Omega^{2}$,

where $R$ is a function of the time coordinate $t$ and the radial coordinate $r, E(r)$ is an arbitrary function of $r$, and $R_{, r}=$ $\partial_{r} R(t, r)$. We get from the Einstein field equations

$$
\begin{aligned}
& \left(\frac{\dot{R}}{R}\right)^{2}=\frac{2 E(r)}{R^{2}}+\frac{2 M(r)}{R^{3}}+\frac{\Lambda}{3}, \\
& \rho(t, r)=\frac{2 M, r}{R^{2} R, r},
\end{aligned}
$$

where $M(r)$ is an arbitrary function of $r$ which arises in the integration of one of the Einstein equations with respect to time, $\dot{R}=\partial_{t} R(t, r)$, and we assume $c=8 \pi G=1$.

The derivation of the analytical solution [15] is based on the introduction of a new coordinate $\eta=\eta(t, r)$ and a variable $a$ by 


$$
\left(\frac{\partial \eta}{\partial t}\right)_{r}=\frac{r}{R} \equiv \frac{1}{a}
$$

and new functions by

$\rho_{0}(r) \equiv \frac{6 M(r)}{r^{3}}, \quad k(r) \equiv-\frac{2 E(r)}{r^{2}}$.

We then express Eq. (2) in the form

$$
\left(\frac{\partial a}{\partial \eta}\right)^{2}=-k(r) a^{2}+\frac{\rho_{0}(r)}{3} a+\frac{\Lambda}{3} a^{4},
$$

where $a$ is now a function of $\eta$ and $r, a=a(\eta, r)$. The coordinate $\eta$, which can be considered a generalization of the conformal time in a homogeneous FLRW universe, is defined implicitly by Eq. (4). The relation between $t$ and $\eta$ is

$t(\eta, r)=\int_{0}^{\eta} a(x, r) \mathrm{d} x+t_{b}(r)$,

which can be computed analytically, and which involve elliptic integrals of the third kind [16].

The function $t_{b}(r)$ is a constant of integration, also called the bang function, since at time $t=t_{b}(r)$ we have $a\left(t_{b}(r), r\right)=0$. This corresponds to the possibility that the big bang can happen at different times at different positions from the center in a LTB space. Its gradient is related to the decaying modes of an early universe density perturbation, and which $\mathrm{CMB}$ observations strongly constrain to be small. In the rest of this paper we will consider a homogeneous big bang, i.e. we will have

$t_{b}(r)=0$,

which in terms of early universe cosmological perturbations corresponds to growing modes only. The solution is given by

$a(\eta, r)=\frac{\rho_{0}(r)}{3 \phi\left(\frac{\eta}{2} ; g_{2}(r), g_{3}(r)\right)+k(r)}$,

where $\phi\left(x ; g_{2}, g_{3}\right)$ is the Weierstrass elliptic function, which satisfies the differential equation

$$
\left(\frac{\mathrm{d} \phi}{\mathrm{d} x}\right)^{2}=4 \phi^{3}-g_{2} \phi-g_{3}
$$

and

$g_{2}=\frac{4}{3} k(r)^{2}, \quad g_{3}=\frac{4}{27}\left(2 k(r)^{3}-\Lambda \rho_{0}(r)^{2}\right)$.

In this paper we will choose the so called FLRW gauge, i.e. the coordinate system in which $\rho_{0}(r)$ is constant. It is convenient to write the solution in terms of dimensionless quantities [17]:

$$
\begin{aligned}
k(r) & =\left(a_{0} H_{0}\right)^{2} K(r), \\
\eta & =T\left(a_{0} H_{0}\right)^{-1}, \\
\rho_{0}(r) & =3 \Omega_{M}^{0}(r) a_{0}^{3} H_{0}^{2}, \\
\Lambda & =3 \Omega_{\Lambda} H_{0}^{2}, \\
a(\eta, r) & =a\left(T\left(a_{0} H_{0}\right)^{-1}, r\right)=\tilde{a}(T, r),
\end{aligned}
$$

to obtain

$$
\begin{aligned}
\tilde{a}(T, r) & =\frac{3 a_{0} \Omega_{M}^{0}(r)}{K(r)+12 \tilde{\phi}\left(T, g_{2}(r), g_{3}(r)\right)}, \\
g_{2}(r) & =\frac{K(r)^{2}}{12}, \\
g_{3}(r) & =\frac{1}{432}\left(2 K(r)^{3}-27 \Omega_{\Lambda}\left(\Omega_{M}^{0}(r)\right)^{2}\right) .
\end{aligned}
$$

We relate the solution expressed in the two different forms by multiplying every term by $\left(a_{0} H_{0}\right)^{2}$ and using the original dimensionful quantities $\eta, k(r), \rho_{0}(r)$ :

$a(\eta, r)=\frac{\rho_{0}(r)}{k(r)+12 \phi\left(\eta, g_{2}(r), g_{3}(r)\right)}=\tilde{a}(T, r)$,

$\phi(\eta, r)=\tilde{\phi}\left(\eta\left(a_{0} H_{0}\right), r\right)\left(a_{0} H_{0}\right)^{2}=\tilde{\phi}(T, r)\left(a_{0} H_{0}\right)^{2}$.

In this form $H_{0}$ is an arbitrary scale which we set equal to the observed value, which will also coincide with the $H_{0}^{\mathrm{LTB}}$ by appropriately setting the value of $T_{0}$ as explained in more detail in [17]. Without any loss of generality we can choose a coordinate system in which $\rho_{0}(r)=$ const., implying that $\Omega_{M}^{0}(r)=$ const., which we will simply denote as $\Omega_{M}$ in the rest of the paper.

\section{Geodesic equations and luminosity distance}

We will solve [18] the null geodesic equation written in terms of the coordinates $(\eta, r)$. We then perform a local expansion of the solution around $z=0$ corresponding to the point $\left(t_{0}, 0\right)$, or equivalently $\left(\eta_{0}, 0\right)$, where $t_{0}=t\left(\eta_{0}, 0\right)$.

The luminosity distance for an observer located at the center of a LTB space-time is given by

$D_{\mathrm{L}}(z)=(1+z)^{2} R(t(z), r(z))=(1+z)^{2} r(z) a(\eta(z), r(z))$,

where $(t(z), r(z))$ or $((\eta(z), r(z))$ is the solution of the null radial geodesic equations as a function of $z$. The equation for geodesics can easily be obtained in the coordinates $(t, r)$,

$\frac{\mathrm{d} t}{\mathrm{~d} r}=-\frac{R_{, r}(t, r)}{\sqrt{1+2 E(r)}}$,

where $t=T(r)$ is the time coordinate along the light-like radial geodesic as a function of the coordinate $r$. Using the definition of the redshift and by following the evolution of a short time interval along the null geodesic $T(r)$, from Eq. (23) we get [19] 


$$
\begin{aligned}
\frac{\mathrm{d} r}{\mathrm{~d} z} & =\frac{\sqrt{1+2 E(r(z))}}{(1+z) \dot{R}_{, r}[r(z), t(z)]}, \\
\frac{\mathrm{d} t}{\mathrm{~d} z} & =-\frac{R_{, r}[r(z), t(z)]}{(1+z) \dot{R}_{, r}[r(z), t(z)]} .
\end{aligned}
$$

We now [18] express the above geodesics equations in the coordinates $(\eta, r)$ :

$$
\begin{aligned}
& \frac{\mathrm{d} \eta}{\mathrm{d} z}=-\frac{\partial_{r} t(\eta, r)+F(\eta, r)}{(1+z) \partial_{\eta} F(\eta, r)} \equiv p(\eta, r), \\
& \frac{\mathrm{d} r}{\mathrm{~d} z}=\frac{a(\eta, r)}{(1+z) \partial_{\eta} F(\eta, r)} \equiv q(\eta, r),
\end{aligned}
$$

where derivation of the expansion of $t(\eta, r)$, while in previous work the coefficients were not evaluated explicitly in terms of $K_{i}$. As a consequence the formulas we obtain only depend on $K_{i}$, and they do not require any addition calculation.

We now find a local Taylor expansion in redshift for the geodesics equations [9], and we then calculate the luminosity distance. The general expression is rather cumbersome, and is given in the appendix. Here we will report only the result assuming $K_{0}=0$, which still shows the general nature of the effect. From a physical point of view fixing $K_{0}$ does not affect the value of $H_{0}$, but it does affect the age of the Universe as shown in [17]. Yet, using the freedom in the choice

$F(\eta, r) \equiv \frac{R_{, r}}{\sqrt{1+2 E(r)}}=\frac{1}{\sqrt{1-k(r) r^{2}}}\left[\partial_{r}(a(\eta, r) r)-a^{-1} \partial_{\eta}(a(\eta, r) r) \partial_{r} t(\eta, r)\right]$.

where the functions $p, q, F$ have explicit analytical forms, making them particularly suitable to derive analytical results.

\section{Formula for the luminosity distance}

In order to obtain the redshift expansion of the luminosity distance we need to expand the relevant functions:

$k(r)=\left(a_{0} H_{0}\right)^{2} K(r)=K_{0}+K_{1} r+K_{2} r^{2}+\cdots$.

We will use Eq. (7) to obtain the expansion for $t(\eta, r)$ from the exact solution for $a(\eta, r)$.

After integration we obtain

$$
\begin{aligned}
& t(\eta, r)=\int_{0}^{\eta_{0}} a(x, r) \mathrm{d} x+a\left(\eta_{0}, r\right)\left(\eta-\eta_{0}\right) \\
& +\frac{1}{2} a^{\prime}\left(\eta_{0}, r\right)\left(\eta-\eta_{0}\right)^{2}+\frac{1}{6} a^{\prime \prime}\left(\eta_{0}, r\right)\left(\eta-\eta_{0}\right)^{3}+\cdots .
\end{aligned}
$$

Using the expression above we obtain the expansion of $t(\eta, r)$ directly from the expansion of $a(\eta, r)$, except for the first term which involves the integral of an elliptic function. The expansion with respect to the radial coordinate $r$ is straightforward and we will not report here all the intermediate results but only the final expression for the solution of the geodesics equations. In this paper we provide the first of the bang function, it is possible to obtain any age, by appropriately fixing it to a constant value $t_{b}(r)=t_{0}$, while since $t_{b}^{\prime}(r)=0$ there would not be any problem related with the compatibility with early universe perturbations which should not contain decaying modes.

We will expand the solution of the geodesic equations according to

$$
\begin{aligned}
r(z) & =r_{1} z+r_{2} z^{2}+\cdots, \\
\eta(z) & =\eta_{1} z+\eta_{2} z^{2}+\cdots, \\
K(z) & =K_{1} z+K_{2} z^{2}+\cdots .
\end{aligned}
$$

After substituting in the geodesics equation we can map the solution of the system of differential equations into a system of algebraic equations for the coefficients of the above expansions. The general expression is rather long and complicated; therefore, here we will report the much simpler case when $K_{0}=0$, while in the appendix we give more general formulas. The motivation for considering the $K_{0}$ case is to focus on the effects of the inhomogeneities which are captured by $K_{1}, K_{2}$, while $K_{0}$ corresponds to the homogeneous component of the curvature function, which in the absence of inhomogeneities is simply the curvature of a FLRW model, and as such it is not associated to any physical effect not already known from standard cosmology.

For the geodesics we get

$$
\begin{aligned}
\eta_{1}= & -\frac{K_{1}\left(T_{0}-1\right) T_{0}+3 \Omega_{M}}{3 a_{0} H_{0} \Omega_{M}}, \\
\eta_{2}= & \frac{1}{\left.36 a_{0} H_{0} \Omega_{\Lambda} \Omega_{M}^{2}\right)}\left[3 \Omega_{\Lambda} \Omega_{M}\left(9 \Omega_{M}^{2}-4 K_{2}\left(-1+T_{0}\right) T_{0}\right)+3 K_{1} \Omega_{\Lambda} \Omega_{M}\left(-4+\left(4-9 \Omega_{M}\right) T_{0}\right.\right. \\
& \left.+\left(-4+9 \Omega_{M}\right) T_{0}^{2}\right)+K_{1}^{2} T_{0}\left(2 \Omega_{\Lambda}\left(2+\left(-4+3 \Omega_{M}\right) T_{0}-6\left(-1+\Omega_{M}\right) T_{0}^{2}+3\left(-1+\Omega_{M}\right) T_{0}^{3}\right)\right. \\
& \left.\left.-4\left(-1+T_{0}\right) W Z+\Omega_{M}\left(-4+T_{0}+3 T_{0} W Z\right)\right)\right],
\end{aligned}
$$




$$
\begin{aligned}
r_{1}= & \frac{1}{a_{0} H_{0}}, \\
r_{2}= & -\frac{1}{12 a_{0} H_{0} \Omega_{M}}\left[\left(9 \Omega_{M}^{2}+K_{1}\left(-4+\left(4-6 \Omega_{M}\right) T_{0}+\left(-4+6 \Omega_{M}\right) T_{0}^{2}\right)\right],\right. \\
r_{3}= & \frac{K_{1}^{2}}{72 a_{0} H_{0} \Omega_{\Lambda} \Omega_{M}^{2}}\left[2 \Omega _ { \Lambda } \left(6\left(3 \Omega_{M}^{2}-4 \Omega_{M}+1\right) T_{0}^{4}-12\left(3 \Omega_{M}^{2}-4 \Omega_{M}+1\right) T_{0}^{3}+3\left(6 \Omega_{M}^{2}-13 \Omega_{M}+4\right) T_{0}^{2}\right.\right. \\
& \left.+2\left(9 \Omega_{M}-4\right) T_{0}+4\right)+3 \Omega_{M}^{2} T_{0}\left(3 T_{0} \zeta_{0}+T_{0}-4\right)-2 \Omega_{M}\left(9 T_{0}^{2} \zeta_{0}+T_{0}^{2}-6 T_{0} \zeta_{0}-4 T_{0}\right. \\
& \left.\left.+6 \zeta_{0}-2\right)+8\left(T_{0}^{2}-T_{0}+1\right) \zeta_{0}\right)+12 K_{1} \Omega_{\Lambda} \Omega_{M}^{2}\left(\left(9 \Omega_{M}-8\right) T_{0}^{2}+\left(8-9 \Omega_{M}\right) T_{0}-5\right) \\
& \left.\left.+3 \Omega_{\Lambda} \Omega_{M}\left(K_{2}\left(\left(8-12 \Omega_{M}\right) T_{0}^{2}+4\left(3 \Omega_{M}-2\right) T_{0}+8\right)+3\left(9 \Omega_{M}-4\right) \Omega_{M}^{2}\right)\right)\right],
\end{aligned}
$$

where

$$
\begin{aligned}
T_{0}\left(a_{0} H_{0}\right)^{-1} & =\eta_{0}, \\
\zeta_{0} & =\zeta\left(\eta_{0}, g_{2}(0), g_{3}(0)\right),
\end{aligned}
$$

and $\zeta$ is the Weierstrass zeta function satisfying the equation $\frac{\mathrm{d} \zeta\left(z, g_{2}(r), g_{3}(r)\right)}{\mathrm{d} z}=-\phi\left(z, g_{2}(r), g_{3}(r)\right)$. show at first and second order, respectively, for $\eta(z)$ and $r(z)$. Contrary to the case of a vanishing cosmological constant, $T_{0}$ now appears explicitly in the formula. This is due to the fact that in a LTB model without cosmological constant it is possible to express explicitly $T_{0}$ in terms of $K_{0}$ and $q_{0}$, the central value of the deceleration parameter, while in our case we have

$$
\begin{aligned}
q_{0} & =-\frac{\ddot{a}\left(t_{0}, 0\right) a\left(t_{0}, 0\right)}{\dot{a}\left(t_{0}, 0\right)^{2}},=\frac{3 \Omega_{M}}{2}-K_{0}-1 \\
& =-\frac{a_{0}^{6} H_{0}^{6}\left(K_{0}^{3}-54 \Omega_{\Lambda} \Omega_{M}^{2}\right)+9 a_{0}^{4} H_{0}^{4} K_{0}^{2} \phi_{0}+27 a_{0}^{2} H_{0}^{2} K_{0} \phi_{0}^{2}+27 \phi_{0}^{3}}{2 a_{0}^{6} H_{0}^{6}\left(2 K_{0}^{3}-27 \Omega_{\Lambda} \Omega_{M}^{2}\right)+18 a_{0}^{4} H_{0}^{4} K_{0}^{2} \phi_{0}-54 \phi_{0}^{3}},
\end{aligned}
$$

The presence of this last function in the formulas obtained above is due to the fact that the function $t(\eta, r)$, which enters the geodesics equation is the integral of $a(\eta, r)$ and depends on $\phi(z)$, has an integral that will depend on $\zeta(z)$. In the case of a LTB solution without a cosmological constant this integral can be performed without the introduction of a new function, while in this case it requires the introduction of $\zeta_{0}$ in the final formula.

The procedure to reduce the analytical formula to this form is rather complicated since it involves the need to express wherever possible all the intermediate expressions in terms of physically meaningful quantities and to use the properties of the elliptic functions. We give more details in the appendix. We see that the effects of inhomogeneities start to where we have used the relations reported in the appendix to simplify the expression, and we have

$$
\begin{aligned}
t_{0} & =t\left(\eta_{0}, 0\right), \\
\phi_{0} & =\phi\left(\eta_{0}, g_{2}(0), g_{3}(0)\right) .
\end{aligned}
$$

Such a relation implicitly constrains the value of $\phi_{0}$ in terms of cosmological parameters but it is not very useful to explicitly determine $T_{0}$, since it would involve to solve a cubic equation first and then to apply the inverse of an elliptic function, while in the vanishing cosmological constant case there exists a simple analytical relation because the Weierstrass function reduces to a trigonometric expression, as shown in [17].

After substituting in the formula for the luminosity distance and expanding we finally get

$$
\begin{aligned}
D_{\mathrm{L}}^{\Lambda \mathrm{LTB}}(z) & =(1+z)^{2} r(z) a^{\Lambda \mathrm{LTB}}(\eta(z), r(z))=D_{1}^{\Lambda \mathrm{LTB}} z+D_{2}^{\Lambda \mathrm{LTB}} z^{2}+D_{3}^{\Lambda \mathrm{LTB}} z^{3}+\cdots \\
D_{1}^{\Lambda \mathrm{LTB}} & =\frac{1}{H_{0}} \\
D_{2}^{\Lambda \mathrm{LTB}} & =-\frac{1}{4 H_{0}}\left(-4+3 \Omega_{M}+2 K_{1}\left(-1+T_{0}\right) T_{0}\right)
\end{aligned}
$$




$$
\begin{aligned}
D_{3}^{\Lambda \mathrm{LTB}}= & \frac{1}{24 H_{0} \Omega_{\Lambda} \Omega_{M}}\left[K _ { 1 } ^ { 2 } \left(2 \Omega_{\Lambda} T_{0}\left(\left(6 \Omega_{M}-5\right) T_{0}^{3}-2\left(6 \Omega_{M}-5\right) T_{0}^{2}+6\left(\Omega_{M}-1\right) T_{0}+2\right)\right.\right. \\
& \left.+\Omega_{M} T_{0}\left(3 T_{0} \zeta_{0}+T_{0}-4\right)-4\left(T_{0}^{2} \zeta_{0}-T_{0} \zeta_{0}+\zeta_{0}-1\right)\right) \\
& +4 K_{1} \Omega_{\Lambda} \Omega_{M}\left(\left(9 \Omega_{M}-8\right) T_{0}^{2}+\left(8-9 \Omega_{M}\right) T_{0}-2\right) \\
& \left.+3 \Omega_{\Lambda} \Omega_{M}\left(-4 K_{2}\left(T_{0}-1\right) T_{0}+9 \Omega_{M}^{2}-10 \Omega_{M}\right)\right]
\end{aligned}
$$

where we used the Einstein equation at the center, $(\eta=$ $\left.\eta_{0}, r=0\right)$

$1=\Omega_{k}(0)+\Omega_{M}+\Omega_{\Lambda}=-K_{0}+\Omega_{M}+\Omega_{\Lambda}$,

$\Omega_{k}(r)=-\frac{k(r)}{H_{0}^{2} a_{0}^{2}}$,

$\Omega_{M}=\frac{\rho_{0}}{3 H_{0}^{2} a_{0}^{3}}$,

$\Omega_{\Lambda}=\frac{\Lambda}{3 H_{0}^{2}}$.

and $T_{0}=\eta_{0}\left(a_{0} H_{0}\right)$ is determined numerically by imposing the conditions [17]

$H^{\mathrm{LTB}}=\frac{\partial_{t} a(t, r)}{a(t, r)}=\frac{\partial_{\eta} a(\eta, r)}{a(\eta, r)^{2}}=\left(a_{0} H_{0}\right) \frac{\tilde{a}^{\prime}(T, r)}{a(T, r)^{2}}$,

$a\left(\eta_{0}, 0\right)=a_{0}$,

$H^{\mathrm{LTB}}\left(\eta_{0}, 0\right)=H_{0}$.

Finally we observe that all the above formulas reduce to the well known FLRW form in the homogeneous limit limit, i.e. when $\left\{K_{1}=K_{2}=0\right\}$.

\section{Testing the accuracy of the formula}

In order to verify the accuracy of the formula obtained we consider the example of an inhomogeneity described by

$K(r)=\epsilon\left(1+r+r^{2}\right)$,

where $\epsilon$ parameterizes the deviation from a homogeneous cosmological model. We then compute the corresponding luminosity distance by integrating numerically the Einstein equations and the geodesic equations, and we compare the numerical results to the redshift expansion for different values of $\epsilon$ (Fig. 1).

As can be seen in the figure the formula is quite accurate up to a redshift of 0.2 , where according to the value of $\epsilon$ the percentual error is approximately between 0.3 and $0.7 \%$. The one provided here is only an example to give a preliminary test of the accuracy of the formula, and as such it does not have any direct connection with the actual size of an inhomogeneity which may be surrounding us. We will investigate more extensively in a separate upcoming paper the range of applicability of the formula in relation with observational

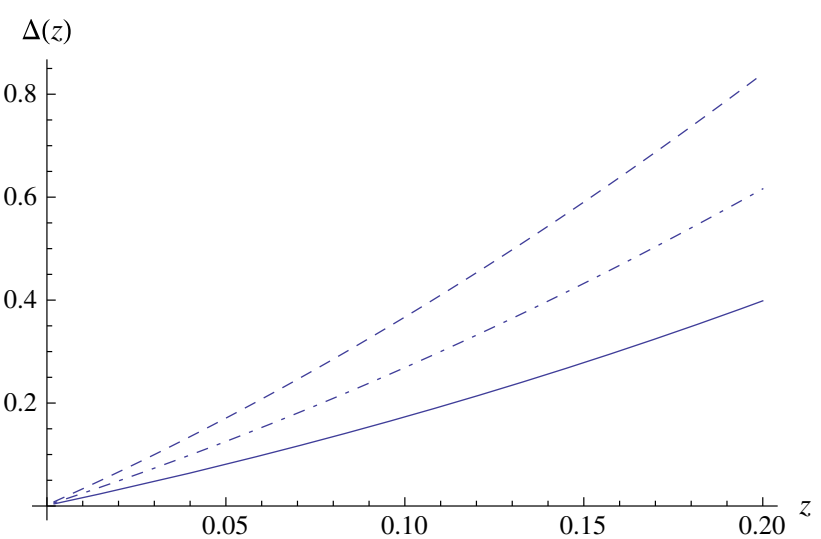

Fig. 1 The percentual error $\Delta=100 \frac{D_{\text {num }}^{\mathrm{LTB}}-D_{\text {Taylor }}^{\mathrm{LTB}}}{D_{\mathrm{num}}^{\mathrm{LTB}}}$ between the numerically computed $D_{\mathrm{Num}}^{\mathrm{LTB}}(z)$ and the Taylor third order expansion $D_{\text {Taylor }}^{\mathrm{LTB}}(z)$ is plotted as a function of the redshift for the LTB solution corresponding to $K(r)=\epsilon\left(1+r+r^{2}\right)$. The solid line corresponds to $\epsilon=0.075$, the dot-dashed line to $\epsilon=0.05$ and the dashed line to $\epsilon=0.1$

data fitting. We also report the percentual error of the formula for the radial coordinate $r(z)$ as a function of the redshift. It is important to observe that $r(z)$ depends on our choice of coordinates, which is $\rho_{0}(r)=$ const., but it is still useful to check its accuracy since it is used in the derivation of the formula for the luminosity distance. This latter one is a physical observable and so its relation with the redshift is independent of our coordinate choice, except for the fact that the coefficients of the Taylor expansion of $K(r)$ would change if we would choose another coordinate system (Fig. 2).

\section{Conclusion}

We have derived the analytical low-redshift expansion of the luminosity distance for a central observer at the center of a spherically symmetric matter inhomogeneity in the presence of a cosmological constant. We have first solved the null radial geodesic equation and calculated the local redshift for $r(z)$ and $\eta(z)$, and we have then used these to calculate the expansion of the luminosity distance. The formulas obtained take a simpler form in the case in which $K_{0}=0$, while in general they are rather long and complicated, but they can be reduced to a more tractable form in the limit in which the deviation from homogeneity can be treated perturbatively.

The formulas we have derived can be used to understand the physical effects of local inhomogeneities in the presence 


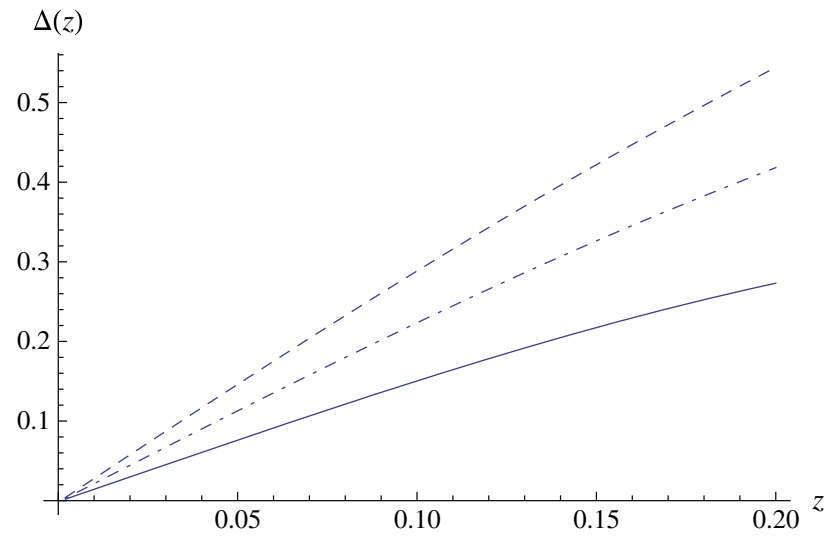

Fig. 2 The percentual error $\Delta=100 \frac{r_{\text {num }}^{\mathrm{LTB}}-r_{\text {Taylor }}^{\mathrm{LTB}}}{r_{\text {num }}^{\mathrm{LTB}}}$ between the numerically computed $r_{\text {Num }}^{\mathrm{LTB}}(z)$ and the Taylor third order expansion $r_{\text {Taylor }}^{\mathrm{LTB}}(z)$ is plotted as a function of the redshift for the LTB solution corresponding to $K(r)=\epsilon\left(1+r+r^{2}\right)$. The solid line corresponds to $\epsilon=0.075$, the dot-dashed line to $\epsilon=0.05$, and the dashed line to $\epsilon=0.1$

of a cosmological constant. It has the advantage, contrary to previous numerical studies, of not depending on any functional ansatz for the profile of the local inhomogeneity. This makes it particularly useful to study possible low-redshift inhomogeneities in a model independent way in the regime in which perturbation theory cannot be applied.

Acknowledgments Chen and Romano are supported by the Taiwan NSC under Project No. NSC97-2112-M-002-026-MY3, by Taiwan's National Center for Theoretical Sciences (NCTS). Chen is also supported by the US Department of Energy under Contract No. DE-AC0376SF00515. AER is also supported by the UDEA Dedicacion Exclusiva and GFIF Sostenibilidad program, and the CODI project IN10219CE.

Open Access This article is distributed under the terms of the Creative Commons Attribution License which permits any use, distribution, and reproduction in any medium, provided the original author(s) and the source are credited.

Funded by $\mathrm{SCOAP}^{3}$ / License Version CC BY 4.0.

\section{Appendix A: Derivation of the analytical formulas}

In order to obtain the formula for $D_{\mathrm{L}}(z)$ in the form in which we reported it in the previous sections we need to apply several simplifying procedures.
The ideas is to express everything in terms of physical quantities, so we start from the definition of $a_{0}$ and $H_{0}$ :

$$
\begin{aligned}
& a_{0}=a\left(\eta_{0}, 0\right)=\frac{\rho_{0}}{k_{0}+3 \phi_{0}}, \\
& H_{0}=\frac{\dot{a}\left(t_{0}, 0\right)}{a\left(t_{0}, 0\right)}=-\frac{3 \phi_{0}^{\prime}}{2 \rho_{0}},
\end{aligned}
$$

where

$\phi_{0}=\phi\left(\eta_{0}, g_{2}(0), g_{3}(0)\right)$,

$\phi_{0}^{\prime}=\left.\frac{\partial \phi\left(\eta, g_{2}(0), g_{3}(0)\right)}{\partial \eta}\right|_{\eta=\eta_{0}}$,

and the derivatives with respect to the time variable $t$ are obtained in terms of derivatives with respect to $\eta$ using Eq. (4). After inverting the above relations we get

$\phi_{0}=\phi\left(\eta_{0}, g_{2}(0), g_{3}(0)\right)=\frac{\rho_{0}-a_{0} k_{0}}{3 a_{0}}$,

$\phi_{0}^{\prime}=\left.\frac{\phi\left(\eta, g_{2}(0), g_{3}(0)\right)}{\mathrm{d} \eta}\right|_{\eta=\eta_{0}}=-\frac{2 H_{0} \rho_{0}}{3}$.

We then substitute the above expressions everywhere where $\left\{\phi_{0}, \phi_{0}^{\prime}\right\}$ appear, which is the reason why they are not present in the formulas obtained.

Another useful relation to simplify intermediate results is the one which can be obtained from the differential equation defining the Weierstrass elliptic function:

$\tilde{\phi}_{0}^{\prime}=\sqrt{-\frac{K_{0}^{3}}{216}-\frac{K_{0}^{2} \tilde{\phi}_{0}}{12}+\frac{\Omega_{\Lambda}\left(\Omega_{M}^{0}\right)^{2}}{16}+4 \tilde{\phi}_{0}^{3} .}$

It can also be shown that the above relation is equivalent to the Einstein equation at the center, $\left(\eta_{0}, 0\right)$,

$1=-K_{0}+\Omega_{\Lambda}+\Omega_{M}$,

since solving the Einstein's equation is reduced to solving the Weierstrass equation by construction [17].

\section{Appendix B: General formulas and perturbative limit}

In this appendix we give the formulas when $K_{0}$ is not zero.

For $\eta(z)$ and $r(z)$ we have

$$
\begin{aligned}
r_{2}= & \frac{1}{1944 a_{0} H_{0} \Omega_{\Lambda} \Omega_{M}^{2}\left(4 K_{0}^{3}-27 \Omega_{\Lambda} \Omega_{M}^{2}\right)}\left[-128 K_{0}^{6} K_{1}\left(T_{0}-1\right) T_{0}^{2}+64 K_{0}^{5} K_{1} T_{0}\left(\left(3 \Omega_{M}-2\right) T_{0}^{2}\right.\right. \\
& \left.+\left(2-3 \Omega_{M}\right) T_{0}-2\right)-972 K_{0}^{4} \Omega_{\Lambda} \Omega_{M}^{2}\left(K_{1} T_{0}^{3}-4\right)+108 K_{0}^{3} \Omega_{\Lambda} \Omega_{M}^{2}\left(27 \Omega_{M}\left(K_{1} T_{0}^{3}-2\right)\right. \\
& \left.-K_{1} T_{0}\left(11 T_{0}^{2}+T_{0}\left(54 \zeta_{0}+34\right)-18\right)\right)-27 K_{0}^{2} K_{1} \Omega_{\Lambda} \Omega_{M}^{2}\left(\left(81 \Omega_{M}^{2}-66 \Omega_{M}+8\right) T_{0}^{3}\right. \\
& -8 T_{0}^{2}\left(\Omega_{M}\left(81 \zeta_{0}+39\right)-81 \zeta_{0}-17\right)+4 T_{0}\left(135 \Omega_{M}-108 \zeta_{0}-34\right) \\
& \left.+144\left(3 \zeta_{0}+1\right)\right)-1458 K_{0} \Omega_{\Lambda} \Omega_{M}^{2}\left(K _ { 1 } \left(2 \Omega _ { M } \left(T_{0}^{2}\left(6 \Omega_{\Lambda}-9 \zeta_{0}-1\right)\right.\right.\right. \\
& \left.\left.\left.+T_{0}\left(-6 \Omega_{\Lambda}+6 \zeta_{0}+4\right)-6 \zeta_{0}+2\right)+3 \Omega_{M}^{2} T_{0}\left(3 T_{0} \zeta_{0}+T_{0}-4\right)+8\left(T_{0}^{2}-T_{0}+1\right) \zeta_{0}\right)+18 \Omega_{\Lambda} \Omega_{M}^{2}\right) \\
& +4374 \Omega_{\Lambda}^{2} \Omega_{M}^{3}\left(K_{1}\left(\left(6 \Omega_{M}-4\right) T_{0}^{2}+\left(4-6 \Omega_{M}\right) T_{0}-4\right)+9 \Omega_{M}^{2}\right]
\end{aligned}
$$




$$
\begin{aligned}
\eta_{1}= & \frac{1}{972 a_{0} H_{0} \Omega_{\Lambda} \Omega_{M}^{2}\left(4 K_{0}^{3}-27 \Omega_{\Lambda} \Omega_{M}^{2}\right)}\left[64 K_{0}^{5} K_{1}\left(T_{0}-1\right) T_{0}^{2}+486 K_{0}^{3} \Omega_{\Lambda} \Omega_{M}^{2}\left(K_{1} T_{0}^{3}-8\right)\right. \\
& -27 K_{0}^{2} K_{1} \Omega_{\Lambda} \Omega_{M}^{2} T_{0}\left(\left(27 \Omega_{M}-4\right) T_{0}^{2}-4 T_{0}\left(27 \zeta_{0}+17\right)+72\right) \\
& \left.-1458 K_{0} K_{1} \Omega_{\Lambda} \Omega_{M}^{2} T_{0}\left(\Omega_{M}\left(3 T_{0} \zeta_{0}+T_{0}-4\right)-4\left(T_{0}-1\right) \zeta_{0}\right)+8748 \Omega_{\Lambda}^{2} \Omega_{M}^{3}\left(K_{1}\left(T_{0}-1\right) T_{0}+3 \Omega_{M}\right)\right] .
\end{aligned}
$$

After substituting in the formula for the luminosity distance

we get

$$
\begin{aligned}
D_{2}^{\Lambda \text { LTB }}= & \frac{1}{972 a_{0} H_{0} \Omega_{\Lambda} \Omega_{M}^{2}\left(4 K_{0}^{3}-27 \Omega_{\Lambda} \Omega_{M}^{2}\right)}\left[64 K_{0}^{5} K_{1}\left(T_{0}-1\right) T_{0}^{2}+486 K_{0}^{3} \Omega_{\Lambda} \Omega_{M}^{2}\left(K_{1} T_{0}^{3}-8\right)\right. \\
& -27 K_{0}^{2} K_{1} \Omega_{\Lambda} \Omega_{M}^{2} T_{0}\left(\left(27 \Omega_{M}-4\right) T_{0}^{2}-4 T_{0}\left(27 \zeta_{0}+17\right)+72\right) \\
& \left.-1458 K_{0} K_{1} \Omega_{\Lambda} \Omega_{M}^{2} T_{0}\left(\Omega_{M}\left(3 T_{0} \zeta_{0}+T_{0}-4\right)-4\left(T_{0}-1\right) \zeta_{0}\right)+8748 \Omega_{\Lambda}^{2} \Omega_{M}^{3}\left(K_{1}\left(T_{0}-1\right) T_{0}+3 \Omega_{M}\right)\right]
\end{aligned}
$$

We do not report higher order terms because the expressions are extremely long and would not add any physical insight, but we consider the case in which we perturbatively treat the function $K(r) \propto \epsilon$, where $\epsilon$ stands for a small deviation from a flat $\Lambda \mathrm{CDM}$ model.

In this perturbative limit for $\eta(z)$ and $r(z)$ we get

$$
\begin{aligned}
\eta_{1}= & -\frac{1}{a_{0} H_{0}}+\frac{\left(K_{1} T_{0}-K_{1} T_{0}^{2}\right) \epsilon}{3 a_{0} H_{0} \Omega_{M}}+O[\epsilon]^{2}, \\
\eta_{2}= & \frac{3 \Omega_{M}}{4 a_{0} H_{0}}+\frac{1}{12 a_{0} H_{0} \Omega_{M}}\left[-4 K_{1}-6 K_{0} \Omega_{M}+4 K_{1} T_{0}+4 K_{2} T_{0}-9 K_{1} \Omega_{M} T_{0}-4 K_{1} T_{0}^{2}\right. \\
& \left.\left.-4 K_{2} T_{0}^{2}+9 K_{1} \Omega_{M} T_{0}^{2}\right) \epsilon\right]+O[\epsilon]^{2} \\
r_{2}= & -\frac{3 \Omega_{M}}{4\left(a_{0} H_{0}\right)}+\frac{\left(2 K_{1}+3 K_{0} \Omega_{M}-2 K_{1} T_{0}+3 K_{1} \Omega_{M} T_{0}+2 K_{1} T_{0}^{2}-3 K_{1} \Omega_{M} T_{0}^{2}\right) \epsilon}{6 a_{0} H_{0} \Omega_{M}}+O[\epsilon]^{2}, \\
r_{3}= & \frac{-4 \Omega_{M}+9 \Omega_{M}^{2}}{8 a_{0} H_{0}}+\frac{1}{6 a_{0} H_{0} \Omega_{M}}\left[\left(2 K_{2}-5 K_{1} \Omega_{M}-9 K_{0} \Omega_{M}^{2}-2 K_{2} T_{0}+8 K_{1} \Omega_{M} T_{0}+3 K_{2} \Omega_{M} T_{0}\right.\right. \\
& \left.\left.-9 K_{1} \Omega_{M}^{2} T_{0}+2 K_{2} T_{0}^{2}-8 K_{1} \Omega_{M} T_{0}^{2}-3 K_{2} \Omega_{M} T_{0}^{2}+9 K_{1} \Omega_{M}^{2} T_{0}^{2}\right) \epsilon\right]+O[\epsilon]^{2} .
\end{aligned}
$$

Finally substituting in $D_{\mathrm{L}}(z)$ the above expansion we obtain

$$
\begin{aligned}
& D_{2}^{\Lambda \text { LTB }}=\left(\frac{1}{H_{0}}-\frac{3 \Omega_{M}}{4 H_{0}}\right)+\frac{\left(K_{0}+K_{1} T_{0}-K_{1} T_{0}^{2}\right) \epsilon}{2 H_{0}}+O[\epsilon]^{2}, \\
& D_{3}^{\Lambda \mathrm{LTB}}=\frac{-10 \Omega_{M}+9 \Omega_{M}^{2}}{8 H_{0}}+\frac{1}{6 H_{0}}\left[3 K_{0}-2 K_{1}-9 K_{0} \Omega_{M}+8 K_{1} T_{0}+3 K_{2} T_{0}-9 K_{1} \Omega_{M} T_{0}-8 K_{1} T_{0}^{2}\right. \\
& \left.\left.-3 K_{2} T_{0}^{2}+9 K_{1} \Omega_{M} T_{0}^{2}\right) \epsilon\right]+O[\epsilon]^{2} .
\end{aligned}
$$

As can be seen $\zeta_{0}$ is not present in the first order perturbative corrections to a homogeneous universe. 


\section{References}

1. S. Perlmutter et al. [Supernova Cosmology Project Collaboration], Measurements of omega and lambda from 42 high-redshift supernovae. Astrophys. J. 517, 565 (1999). arXiv:astro-ph/9812133

2. A.G. Riess et al. [Supernova Search Team Collaboration], Astron. J. 116, 1009 (1998). arXiv:astro-ph/9805201

3. J.L. Tonry et al. [Supernova Search Team Collaboration], Astrophys. J. 594, 1 (2003). arXiv:astro-ph/0305008

4. R.A. Knop et al. [The Supernova Cosmology Project Collaboration], Astrophys. J. 598, 102 (2003). arXiv:astro-ph/0309368

5. B.J. Barris et al., Astrophys. J. 602, 571 (2004). arXiv:astro-ph/ 0310843

6. A.G. Riess et al. [Supernova Search Team Collaboration], Astrophys. J. 607, 665 (2004). arXiv:astro-ph/0402512

7. C.L. Bennett et al., Astrophys. J. Suppl. 148, 1 (2003). arXiv: astro-ph/0302207

8. D.N. Spergel et al., [WMAP Collaboration], Astrophys. J. Suppl. 170, 377 (2007). arXiv:astro-ph/0603449

9. A.E. Romano, M. Sasaki, A.A. Starobinsky, Eur. Phys. J. C 72 , 2242 (2012). arXiv:1006.4735 [astro-ph.CO]
10. B. Sinclair, T.M. Davis, T. Haugbolle, Astrophys. J. 718, 1445 (2010). arXiv:1006.0911 [astro-ph.CO]

11. A.E. Romano, P. Chen, JCAP 1110, 016 (2011). arXiv: 1104.0730 [astro-ph.CO]

12. G. Lemaitre, Ann. Soc. Sci. Brux. Ser. I Sci. Math. Astron. Phys. A 53, $51(1933)$

13. R.C. Tolman, Proc. Nat. Acad. Sci. 20, 169 (1934)

14. H. Bondi, Mon. Not. R. Astron. Soc. 107, 410 (1947)

15. D. Edwards, Mont. Not. R. Astron. Soc. 159, 51 (1972)

16. P.F. Byrd, M.D. Friedman, Handbook of Elliptic Integrals. Lange, Maxwell and Springer Ltd, London (1954)

17. A.E. Romano, Int. J. Mod. Phys. D 21, 1250085 (2012). arXiv: 1112.1777 [astro-ph.CO]

18. A.E. Romano, M. Sasaki, Gen. Rel. Grav. 44, 353 (2012). arXiv: 0905.3342 [astro-ph.CO]

19. M.N. Celerier, Astron. Astrophys. 353, 63 (2000). arXiv:astro-ph/ 9907206 